\title{
Optical properties of gold nanorods macro-structure: a numerical study
}

\author{
A. Gontier ${ }^{1}$, J. Marae-Djouda ${ }^{2}$, R. Caputo ${ }^{3},{\text { Y. } \text { Madi }^{2} \text {, M. Molinari }}^{4}$, G. Lévêque ${ }^{5}$, P.-M. Adam ${ }^{1}$ and T. Maurer ${ }^{* 1}$ \\ ${ }^{1}$ Laboratoire de Nanotechnologie et d'Instrumentation Optique, Institut Charles Delaunay, ICD CNRS UMR 6281, \\ Université de Technologie de Troyes, CS 42060, 10004 Troyes, France \\ ${ }^{2}$ Ermess, EPF-Ecole d'ingénieurs, 3 bis rue Lakanal, 92330 Sceaux, France \\ ${ }^{3}$ Department of Physics and CNR-NANOTEC, University of Calabria, Arcavacata di Rende, 87036 Cosenza, Italy \\ ${ }^{4}$ Laboratoire de Recherche en Nanosciences, EA 4682, Université de Reims Champagne-Ardenne, 21, rue Clément \\ Ader, 51685 Reims Cedex 2, France \\ ${ }^{5}$ Institut d'Electronique, de Microélectronique et de Nanotechnologie (IEMN, CNRS-8520), Cité Scientifique, \\ Avenue Poincaré, 59652 Villeneuve d'Ascq, France
}

Received February 15, 2017; accepted March 29, 2017; published March 31, 2017

\begin{abstract}
In this contribution, a numerical study of the optical properties of closely-packed gold nanorods was performed. The studied nano-objects are experimentally grown on a tilted polydimethylsiloxane (PDMS) substrate by using physical vapor deposition (PVD). This method creates nanorods tilted to a certain angle with respect to the substrate normal. This geometry allows exciting both transverse and longitudinal modes of the rods. As demonstrated in a previous experimental work, such PVD-grown nano-objects show promising possibilities both as strain gauges or strain-tunable metamaterials if fabricated on a stretchable dielectric substrate. This numerical study is based on experimental data from previous work and pushes further the subject by approaching an optimized nano-structure allowing better strain-sensitivity (particularly by changing the auto-organization of the said nanorods).
\end{abstract}

Gold nanorods, because of their chemical stability and great anisotropic optical properties in the visible range, have been extensively studied over the past few decades. Their application covers topics ranging from optical data storage [1] to controlled gene delivery [2], biological and chemical sensing [3-8] and active plasmonics [9]. In this letter, the optimization of the nanorod characteristics for plasmonic applications is studied through numerical simulations [10]. The following data take advantage of those outstanding properties to design a highly strainsensitive plasmonic system.

PDMS is an ideal substrate for such plasmonic strain sensors because of its stable mechanical and optical behavior during stretching. Besides, it is perfectly transparent to visible light and has an elasticity range allowing wide deformations without plasticity. Figure 1 shows an idealized sketch of the nanorods brush which can be fabricated by using a physical vapor deposition (PVD) technique on a tilted substrate [11]. After deposition, the rods are 100nm long and exhibit diameters of $20 \mathrm{~nm}$ which could be easily simulated.

${ }^{*}$ E-mail: thomas.maurer@utt.fr
In this study, a commercial-grade simulator based on the finite-difference time-domain (FDTD) method was used to perform the calculations [12] and obtain an optical response of the rods to plane wave excitation.

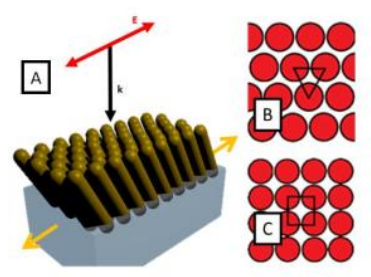

Fig. 1. A) Scheme of the nanorods brush based on SEM images of PVDgrown nanorods onto PDMS [11], B) hexagonal and C) Square array.

To understand the behavior of gold nanorods in an array, the properties of an isolated nanorod on the substrate are firstly analyzed. These calculations allow clarifying the influence of excitation polarization on the response of the system. Thanks to the nanorod tilt, the optical response of a single rod is highly sensitive to the polarization of the incident light. Indeed, if the nanorod was perfectly vertical, only transverse modes could be excited by the incident plane wave. However, a tilted nanorod allows selective excitation of both transverse and longitudinal modes provided that the incident light polarization is along the nanorod long axis (see Fig. 1A and Fig. 2). This is a crucial feature to optimize the sensitivity of the designed plasmonic strain sensors as recently demonstrated in our experimental study on such systems. Figure 2 demonstrates precisely how overriding the longitudinal mode is amplitude-wise, making the $570 \mathrm{~nm}$ transverse mode negligible. The $770 \mathrm{~nm}$ longitudinal mode is thus more adapted to strain monitoring thanks to its great amplitude and narrowness. 


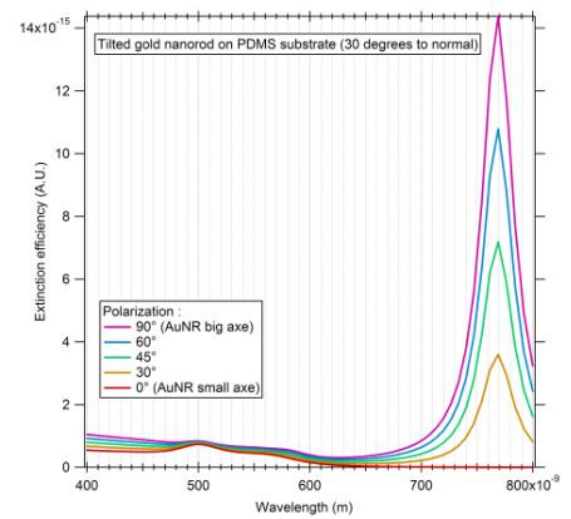

Fig. 2. Transmission spectra of a gold nanorod, tilted by $30^{\circ}$ with respect to the substrate normal, depending on the angle between the incident polarization light and the nanorod long axis. When the light polarization is transverse to the nanorod long axis, the longitudinal mode cannot be excited.

As evidenced in Fig. 1, the system which is here studied consists in an assembly of nanorods which are side-toside. The goal is to understand the variation of the coupling between their plasmon longitudinal modes depending on the distance between them. The initial center-to-center distances are $30 \mathrm{~nm}$ so that the plasmon modes can be considered as initially weakly coupled, referring to the plasmon ruler equation [13]. The incident light and the stretching directions are parallel as shown in Fig. 1A.

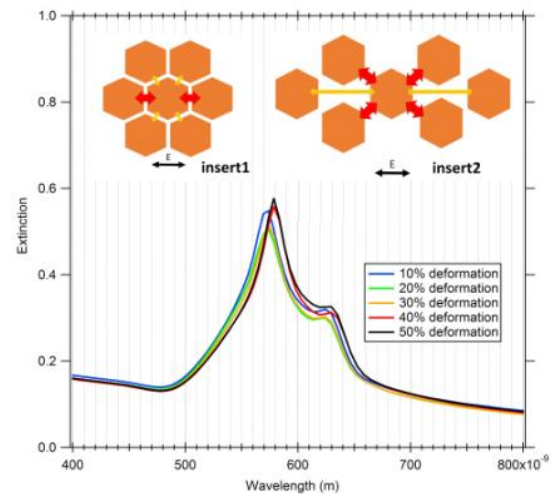

Fig. 3. Transmission spectra calculated for a triangular lattice of $\mathrm{Au}$ nanorods. No visible effect may be observed because during the stretching along the incident light polarization direction, the displacements of the rods make some of them couple while others decouple. The two inserts ( 1 at rest and 2 under strain) provide schematics illustrating a coupling increase between the crossed nanorods.

The first major result concerns the role of the nanorods packing. Indeed, Fig. 3 presents the transmission spectra for triangular lattices of nanorods (see Fig. 1B) when the incident polarization is set along the stretching direction. No visible effect may be seen, due to a phenomenon which could be designed as "cross coupling". For triangular lattices of nanorods stretching the substrate leads to coupling and decoupling of nanorods along the polarization direction at the same time, which tends to hide any effects. Indeed, during the stretching, a compression occurs on the axis transverse to the stretching direction. Therefore, the displacements of the nanorods tend to align the closest neighbors along the stretching direction. This phenomenon induces coupling between the crossed nanorods - as shown in the insert of Fig. 3. This cross coupling compensates for the decoupling of the first neighbors along the polarization axis.
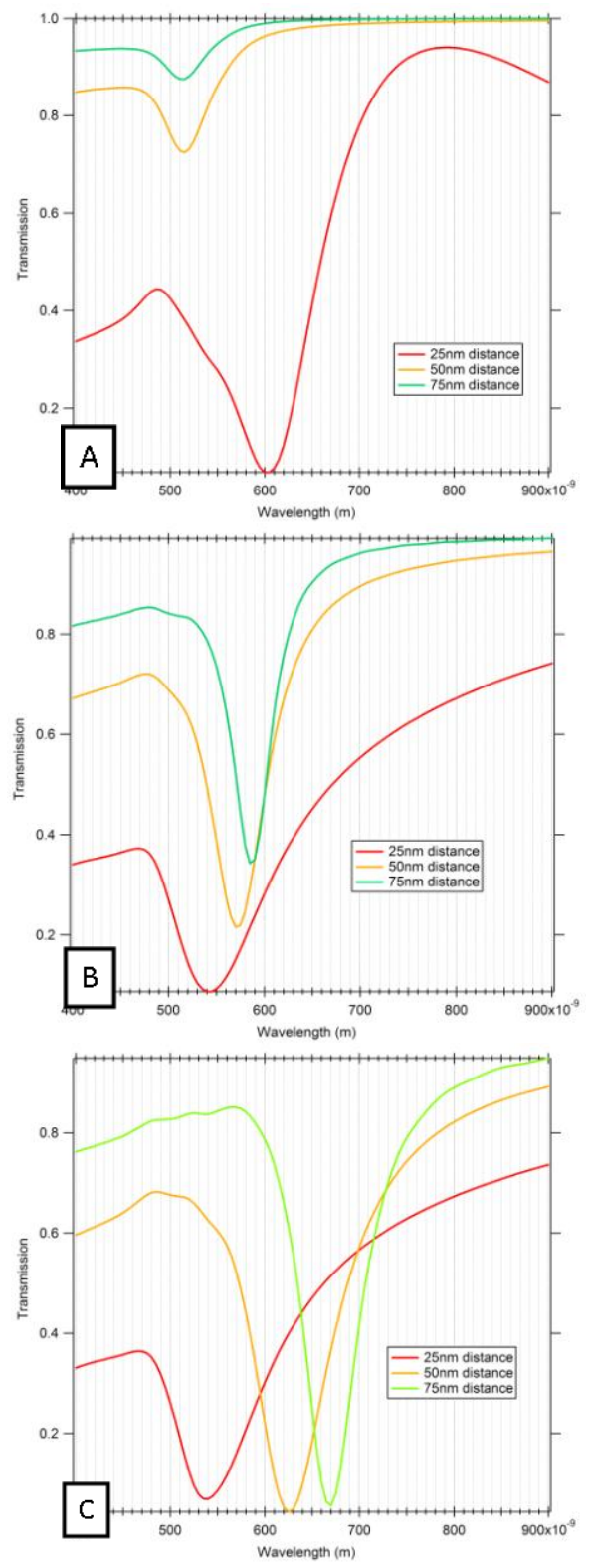

Fig. 4. Transmission spectra of a square lattice of Au nanorods with a $45^{\circ}$ tilt to the substrate normal. a) $50 \mathrm{~nm}$ long nanorods; b) $100 \mathrm{~nm}$ long nanorod; c) $150 \mathrm{~nm}$ long nanorods. The initial center-to-center is $25 \mathrm{~nm}$.

The substrate is stretched so that the center-to-center distance for 
nanorods aligned along the stretching direction increases up to $50 \mathrm{~nm}$ (100\% of deformation) and $75 \mathrm{~nm}$ ( $200 \%$ of deformation).

Therefore, it appears that square lattices of $\mathrm{Au}$ nanorods are more adapted for strain monitoring. However, a key parameter is the length of nanorods and Fig. 4 illustrates its drastic impact on the sensitivity of such systems for strain monitoring. As the length of the nanorods increases, the surface exposed to coupling with neighboring nanorods also increases. This relation is a major indication for upcoming optimizations toward a better strain sensor or a strain-tunable metamaterial. With rods whose length increases from $50 \mathrm{~nm}$ to $150 \mathrm{~nm}$, the spectral shift, in addition to its great increase, even changes the direction from a blueshift to a redshift. Such a drastic change has not been reported in the literature so far. To illustrate this switching behavior, we refer, by electromagnetic analogy, to molecular orbital theory as depicted by Prashant K. Jain et al. [14] and adapt it to our system (see Fig. 5). This analogy is based on similar energetic behavior of plasmon coupling and formation of bonding and anti-bonding modes.

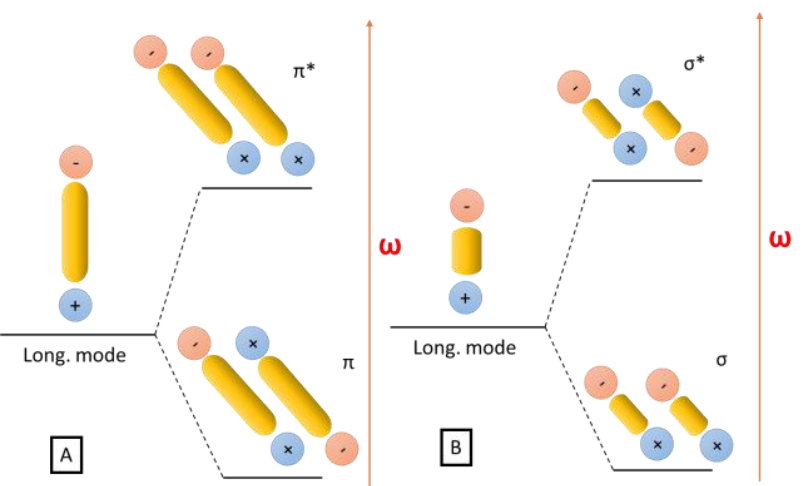

Fig. 5. Energy diagram of the nanorod coupling modes explaining the optical behavior of resonance positions during stretching with a) long nanorods and b) small nanorods

When the nanorods are long enough regarding the considered tilt angle (see Fig. 5A), the high-energy level (mode $\pi^{*}$ ) is bright- exhibiting a non-zero dipolar momentum configuration- and thus is characterized by a red-shift when the nanorods are getting further from each other (see Figs. 4B and C). On the other hand, when the nanorods exhibit a lower aspect ratio, the bright coupling mode corresponds to the lower energy level and in that case, a blueshift will characterize the decoupling. This phenomenon is caused by the predominant transverse mode with respect to longitudinal. These results tend to indicate that for every tilt angle considered, there is a critical nanorod length at which the strain sensitivity will be close to zero.
In conclusion, through numerical simulations on a system of $\mathrm{Au}$ nanorods supported by a stretchable substrate, different configurations were studied to determine the proper characteristics of these nanorod 'brushes' to be used as strain sensitive devices. The first observation is that the PVD nanorods growth in a triangular lattice causes cross coupling which inhibits resonance shifts by the compensation mechanism of transverse compression. Then, depending on the rods aspect ratio, there is a tendency of low aspect ratio nanorods to switch dark and bright modes when passing from low aspect ratios to higher ones. This indication can represent a reliable suggestion to focus on in peculiar fabrication designs. Further studies dedicated to the dark-bright mode switching could bring light on the matter and suggest what is the critical length causing the nullification of strain sensitivity.

The authors are thankful to the "région ChampagneArdenne " and FEDER fundings for the MecaOpt essaimage program. They also thank the DRRT Champagne Ardenne, the "conseil general de l'Aube" and the FEDER for supporting the Nano'Mat platform (www.nanomat.eu). T.M. and R.C. thank the Labex ACTION project (contract ANR-11-LABX-01-01) for financial support and the COST Actions IC1208 and MP1302 for networking support.

\section{References}

[1] J.W.M. Chon, C. Bullen, P. Zijlstra, M. Gu, Adv. Funct. Mater. 17, 875 (2007).

[2] C.-C. Chen, Y.-P. Lin, C.-W. Wang, H.-C. Tzeng, C.-H. Wu, Y.-C. Chen, C.-P. Chen, L.-C. Chen, Y.-C. Wu, J. Am. Chem. Soc. 128, 3709 (2006).

[3] J.N. Anker, W.P. Hall, O. Lyandres, N.C. Shah, J. Zhao, R.P. Van Duyne, Nat. Mater 7, 442 (2008).

[4] B. Sepulveda, P.C. Angelome, L.M. Lechuga, L.M. Liz-Marzań, Nano Today 4, 244 (2009).

[5] A. Haes, R.P. Van Duyne, J. Am. Chem. Soc. 124, 10596 (2002).

[6] J.C. Riboh, A.J. Haes, A.D. McFarland, C.R. Yonzon, R.P. Van Duyne, J. Phys. Chem. B 107, 1772 (2003).

[7] C.R. Yonzon, E. Jeoung, S. Zou, G.C. Schatz, M. Mrksich, R.P. Van Duyne, J. Am. Chem. Soc. 126, 12669 (2004).

[8] A.J. Haes, L. Chang, W.L. Klein, R.P. Van Duyne, J. Am. Chem. Soc. 127, 2264 (2005)

[9] R. Caputo, G. Palermo, M.Infusino L. De Sio, Nanospectroscopy 1, 40 (2015).

[10] T. Maurer, J. Marae-Djouda, U. Cataldi, A. Gontier, G. Montay, Y. Madi, B. Panicaud, D. Macias, P.-M. Adam, G. Lévêque, T. Bürgi, R. Caputo, Frontiers of Materials Science 9, 170 (2015).

[11] X. Niu, S. P. Stagon, H. Huang, J.K. Baldwin, A. Misra, Phys. Rev. Lett. 110136102 (2013).

[12] Lumerical Solutions, Inc. http://www.lumerical.com/tcad-products/fdtd/

[13] P.K. Jain, W. Huang, M.A.El-Sayed, Nanoletters 7, 2080 (2007).

[14] P.K. Jain, M.A. El-Sayed, Chem. Phys. Letters 487, 153 (2010); doi:10.1016/j.cplett.2010.01.062. 